\title{
Fathoming the kynurenine pathway in migraine: why understanding the enzymatic cascades is still critically important
}

\author{
Martina Curto - Luana Lionetto - Francesco Fazio • \\ Dimos-Dimitrios Mitsikostas • Paolo Martelletti
}

Received: 22 October 2014/ Accepted: 27 January 2015

(C) SIMI 2015

\begin{abstract}
Kynurenine pathway, the quantitatively main branch of tryptophan metabolism, has been long been considered a source of nicotinamide adenine dinucleotide, although several of its products, the so-called kynurenines, are endowed with the capacity to activate glutamate receptors, thus potentially influencing a large group of functions in the central nervous system (CNS). Migraine, a largely unknown pathology, is strictly related to the glutamate system in the CNS pathologic terms. Despite the large number of studies conducted on migraine etio-
\end{abstract}

M. Curto and L. Lionetto equally contributed to the manuscript.

M. Curto

Psychiatric Unit, Neurosciences, Mental Health and Sensory Organs (NESMOS) Department, Sant'Andrea Hospital, Sapienza University of Rome, Rome, Italy

M. Curto

Department of Psychiatry, Harvard Medical School, Boston, MA, USA

L. Lionetto $(\square)$

Advanced Molecular Diagnostics Unit (DiMA), Sant'Andrea

Hospital, Rome, Italy

e-mail: luanalionetto@gmail.com

F. Fazio

I.R.C.C.S. Neuromed, Parco Tecnologico, Pozzilli, IS, Italy

D.-D. Mitsikostas

Neurology Department, AthensNaval Hospital, Athens, Greece

P. Martelletti

Department of Clinical and Molecular Medicine, Sapienza

University of Rome, Rome, Italy

P. Martelletti

Regional Referral Headache Centre, Sant'Andrea Hospital,

Rome, Italy pathology, the kynurenine pathway has been only recently linked to this disease. Nonetheless, some evidence suggests an intriguing role for some kynurenines, and an exploratory study on the serum kynurenine level might be helpful to better understand possible alterations of the kynurenine pathway in patients suffering from migraine.

Keywords Migraine - Kynurenic acid · Kynurenine · Xanthurenic acid · Anthranilic acid · Quinolinic acid

\section{Introduction}

L-Tryptophan is an essential amino acid having in its chemical structure an indole substitute on the $\beta$ carbon. The principal role of tryptophan in humans is as a constituent of protein synthesis, even if it is also the precursor of two important metabolic pathways such as serotonin and kynurenine synthesis [1]. Although serotonin synthesis accounts for approximately $5-10 \%$ of tryptophan catabolism, it has been a topic of intense research given its wellknown action as a neurotransmitter [2, 3]. In particular, serotonergic mechanisms have been involved both in mood disorders and in migraine pathophysiology [4]. In fact, the main pharmacological approach to mood disorders is represented by the selective serotonin reuptake inhibitors (SSRI); whereas triptans, the 5-HT receptors agonists, are considered a first-line treatment in acute migraine [5]. The kynurenine pathway (KP), accounting for more than $90 \%$ of the tryptophan metabolism, has received less attention, even though it generates neuroactive compounds that are able to interact with glutamate receptors in the central nervous system (CNS) [6]. In the past few decades, the role of KP has been intensively studied in several psychiatric disorders as well as in neurodegenerative diseases [7, 8]. 
More recently, scientists report a possible involvement of kynurenine metabolites in migraine pathophysiology [9]. Migraine has a complex pathophysiology in which both central and peripheral components of the trigeminal pain pathway play a central role $[10,11]$. The trigemino-vascular activation during the attack has largely been described, and recently the brainstem nuclei, called "migraine generators", have been reported to be involved in migraine. Moreover, a series of destabilizing events within the brain trigger a cortical spreading depression (CSD), responsible for the aura phenomena and for trigeminal activation [12]. The role of glutamate is heavily supported both in the trigemino-vascular as well as in brainstem nuclei activation, and furthermore in the CSD initiation and propagation [13]. Some of the KP metabolites have been demonstrated to interact both with ionotropic and metabotropic glutamate receptors [6]. Therefore, their possible role in the pathophysiology of migraine will be extensively reviewed in this paper.

\section{The L-tryptophan kynurenine pathway}

The KP accounts for more than $90 \%$ of the essential aminoacid L-tryptophan metabolism in humans, in parallel with serotonin production. The KP of tryptophan metabolism generates neuroactive compounds that are able to interact with glutamate receptors in the CNS [6]. The first step of the pathway is the conversion of tryptophan into $\mathrm{N}$ formylkynurenine, catalyzed by either indolamine-2,3dioxygenase (IDO) or tryptophane-2,3-dioxygenase (TDO) (Fig. 1). Both enzymes are present in the CNS, although their expression levels are lower than in peripheral cells where TDO is mainly expressed in liver [14]. $N$-Formylkynurenine is then converted by formamidase into kynurenine (KYNU), which is hydroxylated into 3-hydroxykynurenine (3-HK) by kynurenine monoxygenase (KMO), or alternatively, transaminated into kynurenic acid (KYNA), by types 1, 2 and 3 kynurenine aminotransferases (KAT1-3), or transformed into anthranilic acid (ANA) by kynurenines. 3-HK is sequentially transformed into 3-hydroxyanthranilic acid (3-HANA), and quinolinic acid (QUINA), or transaminated to xanthurenic acid (XA) [6]. Cinnabarinic acid is a by-product of the pathway, which derives from the condensation of two molecules of 3-HANA [15]. In the last step of the KP, QUINA is converted into the coenzymes nicotinamide adenine dinucleotide (NAD), and NAD phosphate (NADP), by a phosphoribosyltransferase (Fig. 1). The KP enzymatic reactions are located in macrophages, microglial cells and, partially, in astrocytes [16]. KYNA and QUINA have been the subject of extensive investigation since they have been shown to interact with ionotropic glutamate receptors.
QUINA has been found in nanomolar concentrations in the CNS, and acts as an orthosteric agonist at the GluN2 subunits of $N$-methyl-D-aspartate (NMDA) receptors [17]. Moreover, it might regulate endogenous glutamate release and uptake inhibition and lipid peroxidation [18]. In contrast, KYNA acts as a competitive antagonist at the glycine site on the GluN1 subunit of NMDA receptors, thereby inhibiting NMDA receptor function [19]. KYNA also inhibit the kainate (GluK) and the amino-3-hydroxy-5methyl-4-isoxazole propionic acid (AMPA) glutamate receptors (GluR), and has a non-competitive inhibitory action on the $\alpha 7$-nicotinic acetylcholine (nACh) receptors [20]. Similarly to QUINA, it has been found in the CNS at nanomolar concentrations, and exerts a possible neuroprotective effect [21]. It has been demonstrated to reduce the neuronal loss in excitotoxic, ischemia-induced and neuronal injuries [22].

To date, 3-HK and 3-HANA have mainly been studied, since they have been shown to induce neurotoxic effects by increasing oxidative stress and the production of free radicals or through excitotoxicity [23, 24]. In fact, both the molecules autoxidize under physiological conditions producing highly reactive hydroxyl radicals, even if these properties are partially balanced by their related antioxidant capacity to scavenge peroxyl radicals [25]. Moreover, cinnabarinic acid has been reported as an orthosteric agonist of mGlu4 receptors with no activity at other mGlu receptor subtypes endowed with neuroprotective activity [15]. The production of XA from 3-HK lowers the concentration of the latter, reducing its neurotoxicity [26]. Moreover, XA has been recently demonstrated to be a putative agonist of metabotropic glutamate receptors $2 / 3$ (mGlu2/3) [27].

\section{Glutamate relevance in migraine pathophysiology}

Migraine is a common, recurrent, and disabling primary headache disorder affecting up to $20 \%$ of the population with a female/male ratio of 3/1 [28]. The central clinical symptoms of migraine are represented by attacks of unilateral throbbing headache, generally followed by nausea, photophobia and phonophobia, and worsened by physical activity. Before the headache attacks, about onethird of migraineurs experience an aura, a focal neurological disturbance clinically manifested as visual, sensitive or motor symptoms due to a cortical spreading depression (CSD) originating in the occipital cortex [29]. CSD is a slowly progressing wave of depolarization followed by a long-lasting suppression of neuronal activity and excitability propagating through the cortex. The exact physiopathology of migraine is still not fully understood, and the brain events that initiate the migraine attacks 
Fig. 1 The kynurenine pathway. 1.a Indoleaminepyrrole 2,3-dioxygenase; $1 . b$ tryptophan 2,3-dioxygenase; 2 arylamine formamidase; 3 kynurenine aminotransferase; 4 kynurenine 3-hydroxylase; 5 kynureninase; 6

3-hydroxyanthranilic acid 3,4dioxygenase; 7 autoxidation<smiles>N[C@@H](Cc1c[nH]c2ccccc12)C(=O)O</smiles><smiles></smiles><smiles>N[C@@H](CC(=O)c1ccccc1NC=O)C(=O)O</smiles>

N-Formylkynurenine

2

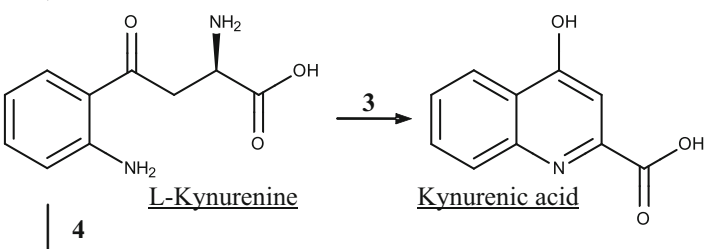<smiles>Nc1ccccc1C(=O)O</smiles>

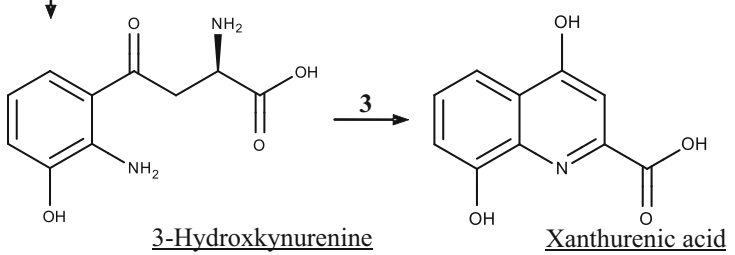

Anthranilic acid<smiles>Nc1c(C(=O)O)c2nc3c(C(=O)O)cccc3oc-2cc1=O</smiles>

Cinnabarinic acid

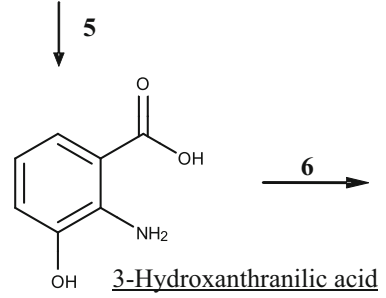<smiles></smiles>

remain unclear. The most well-established and accepted migraine pathophysiological mechanisms involve (1) the peripheral sensitization of the trigemino-vascular system (TS) [11], (2) the central sensitization of the caudal trigeminal nucleus (TNC) [30], as well as the (3) activation of brainstem generator nuclei [31], and (4) the development of the CSD [32]. To be precise, the order of appearance of these phenomena is not necessarily the same as described in the text.

Glutamate plays a key role in the migraine pathophysiological mechanisms, even if studies conducted to compare plasma and platelet glutamate concentrations in migraineurs and in controls lead to conflicting results [13, 33]. Nevertheless, systematically elevated glutamate levels lead to spontaneous headache in humans, and also to significant craniofacial muscle sensitization [34]. The importance of glutamate has been reported at many levels of the nervous system; therefore, the comprehension of its role in migraine might be crucial for the development of novel therapeutic approaches.

1. The peripheral trigeminal nociceptors sensitization results from the following process: the vasodilatation of the dural and pial blood vessels arises and acts as a trigger mechanism, leading to a neuropeptides release from perivascular trigeminal primary nociceptors, thereby inducing sterile neurogenic inflammation [35]. In fact, the released calcitonin gene-related peptide (CGRP), substance $\mathrm{P}$ and neurokinin $\mathrm{A}$ stimulate the trigeminal first-order neurons, leading to their peripheral sensitization [36]. This process is clinically reflected by the throbbing nature of the pain, and its worsening during activities that increase intracranial pressure. The glutamate receptors are 
expressed in the dorsal root, the TG primary sensory afferents and together with the $\alpha 7$-nACh receptors, and are involved in nociceptive signaling [9]. A recent study reports that glutamate dysregulation in the TG is a novel mechanism for peripheral sensitization of the craniofacial region [37]. Similar is the sensitization of craniofacial muscles in the rat, caused by elevated glutamate levels in the TG, is in part NMDA-mediated, and driven by peripheral processes [37].

2. The TNC is located in the medulla and in the superior part of the spinal cord, and receives the central projections of the trigeminal first-order neurons, whose cell bodies are located in the trigeminal ganglion (TG). Conversely, the first-order neurons peripheral projections innervate the intracranial pain structures. The activation of the second-order trigeminal neurons involves glutamate as the main neurotransmitter [30]. Glutamate levels are increased in the TNC after stimulation of the first-order trigeminal neurons, and both the NMDA receptors and other glutamate receptors are expressed in the TNC [38]. NMDA receptors mediate the activation of the second-order neurons, even if other glutamate receptors antagonists contribute to their inhibition [39]. Moreover, even the $\alpha 7$ nACh receptors presynaptically modulate the activation of the second-order neurons and therefore the transmission of nociceptive information to the CNS [40]. The activation of second-order trigeminal neurons leads to the central sensitization of the TNC, clinically revealed by cutaneous allodynia of the head and face [30]. The glutamate receptors are involved in the central sensitization process: the increase in extracellular glutamate levels directly correlates with sensory threshold changes on the face of rats [38].

3. The activation of specific brainstem nuclei, called "migraine generators", has been well demonstrated during a migraine attack [41]. These nuclei belong to the pain regulatory structures, and include the dorsal raphe nucleus (DRN), the nucleus raphe magnus (NRM), the locus coeruleus (LC), and the periaqueductal gray matter (PAG) [42]. Therefore, a loss of nociception regulatory function of the brainstem nuclei may be involved in migraine pathophysiology [43]. In the past few decades, glutamatergic neurotransmission has been reported involved in the activation of migraine generators. In fact, glutamate receptors antagonist reduces the activity of NRM, glutamate levels increase in LC after sciatic nerve or neuronal stimulation in PAG [44].

4. The last potential trigger mechanism of migraine is the CSD, a slowly progressing wave of depolarization followed by a long-lasting suppression of neuronal activity and excitability and accompanied by slowly spreading cortical hypoperfusion. CSD is largely considered the neuronal basis for the aura phenomenon [12]. Although the CSD initiation of a migraine attack process still remains unclear, CSD itself has been demonstrated to initiate the trigeminal sensitization by the activation of the trigemino-vascular afferent and the induction of sterile inflammation (i.e., increased blood flow and protein extravasation) in the dura mater [45]. Glutamate and its receptors perhaps initiate a CSD [46]. Administration of glutamate triggers a CSD and suddenly dilates pial arterioles in the mouse cortex, while NMDA receptor antagonists, but not nonNMDA receptors antagonists, can reduce the number of CSD waves and decrease the permeability of the BBB during CSD [46]. Furthermore, recent data demonstrate that NR2B-containing NMDA receptors are key mediators of CSD, and that selective changes in several glutamate receptors binding sites (NMDA, AMPA, and kainate) are related to the delayed excitatory phase after CSD [47]. Therefore, glutamatergic neurotransmission is also linked to trigeminal activation by its initiation and propagation of the CSD.

\section{KP metabolites in migraine}

\section{L-Kynurenine}

Tryptophan metabolism to L-kynurenine, catalyzed by the $\mathrm{KP}$ rate-limiting enzyme indolamine 2,3-dioxygenase (IDO), is stimulated by pro-inflammatory cytokines such as interferon- $\gamma$ (IFN- $\gamma$ ), and inhibited by interleukin-10 (IL10 ), tumor growth factor- $\beta$ (TGF- $\beta$ ), and IL-4 [48]. As KYNU can cross the blood brain barrier (BBB), and about $60 \%$ of its brain concentration comes from the periphery, peripheral inflammation is likely to increase central KYNU levels [49]. Both systemic and central immune stimulation, including cytokine-induced IDO activation, are believed to contribute to the cerebral KP activation in neurological disorders [6]. Some studies report a decrease in IL-4 serum levels in migraineurs, and an increase in the IL-10 levels $[50,51]$. Moreover, tryptophan depletion is known both to compromise immune responses, and to intensify photophobia and other symptoms in migraine patients [52]. The IDO over-expression stimulated by pro-inflammatory cytokines may result in a decrease of serotonin availability in parallel to an increase of KYNU synthesis [9]. Although serotonin production is deficient in patients suffering from migraine, KYNU itself has been demonstrated to act as a vasodilator activating soluble guanylylcyclase and cyclic GMP (GC-cGMP), adenylyl cyclase and cyclic AMP (ACcAMP) [48, 53]. Therefore, considering the possible 
involvement of IDO activity in migraine pathogenesis, the evaluation of its genetic polymorphisms in patients might help to better elucidate the KP association with migraine. Moreover, the serum KYNU/TRP ratio, used as an indirect index of IDO activity, has never been studied in migraine patients, and might represent a useful tool for the characterization of IDO activity role in migraine [54].

\section{Kynurenic acid}

Kynurenic acid acts in the brain as a glycine-site NMDA receptors competitive antagonist. It also acts at the glutamate site of the NMDA receptors, antagonizes the $\alpha 7$ nACh receptors, and selectively activates the G-protein coupled receptor GPR35 [55]. KYNA neuroprotective and anticonvulsive properties have been reported in animal models of neurodegenerative diseases [48]. KYNA is not transported across the $\mathrm{BBB}$, and its levels in the brain are determined by the local synthesis from KYNU. The transformation is catalyzed by several kynurenine aminotransferases (KATs), of which KAT-II is the principal enzyme producing KYNA, expressed solely in astrocytes [56]. Glial cells further release KYNA in the extracellular compartment. Although the KYNA re-uptake processes have still not been identified, the probenecid-sensitive organic anion transporters 1 and 3 (OAT 1,3) have been involved in its active removal from the extracellular compartment [55]. In migraine, antinociceptive effects of KYNA have been reported in both the first- and the secondorder trigeminal nociceptors. Moreover, KYNA reduces the activation of migraine generators, and inhibits CSD [48]. KYNA antiglutamatergic activity might be the neurobiological basis of the CSD inhibition. In fact, both the NMDA receptor antagonist dizocipiline and KYNA are able to reduce the number of CSD waves, and to decrease the permeability of the BBB during CSD [57]. Moreover, glutamate levels are increased during CSD. KYNA inhibits CSD both in the cerebellum and in the neocortex, and increased KYNA levels are found in rat brain after a triggered CSD [58]. Sole KYNU systemic administration reduces CSD frequency in female rats, whereas in males, the same effect occurs after co-administration of probenecid, the difference regulated by the influence of ovarian hormones [59]. The suppressive effect on CSD is related to an increase in cerebral levels of KYNA. These data suggest that KYNA might offer a new approach for migraine with aura treatment (Fig. 2). KYNA modulates second-order nociceptors in the TS (Fig. 2). In fact, in animal models of migraine, the administration of KYNA or its analogues, and the co-administration of KYNU with probenecid significantly mitigates the central sensitization of the TNC [60-62]. At peripheral nociceptors level, KYNA shows antinociceptive effects as well (Fig. 2). Both the systemic and the local KYNA administration reduce nociception and allodynia [63, 64]. Moreover, KAT expression is reduced in a migraine model such as the electrical stimulation of the trigeminal ganglion (TG) [65]. A KYNA derivative inhibits the activation of the primary sensory fibers, and therefore the subsequent release of CGRP [62]. Beside the peripheral glutamate receptors, two other possible KYNA targets are represented by peripheral $\alpha 7$-nACh receptors and the G-protein-coupled receptor-35 (GPR35) (Fig. 2), involved in nociceptive signaling [66, 67]. Fejes-Szabó et al. [67] suggest that a KYNA derivative can dose-dependently modulate the changes in neurochemical markers of activation and sensitization of the trigeminal system, possibly acting on peripheral and central glutamate or $\alpha 7$-nicotinic acetylcholine receptors. Independently of its biochemical actions at receptors, KYNA also shows antioxidant features, which are linked to its ability to scavenge hydroxyl, superoxide anion and other free radicals [68]. Therefore, KYNA and its derivatives represent a novel and useful therapeutic approach to migraine pharmacological treatment.

\section{3-Hydroxykynurenine}

Kynurenine is oxidized into 3-HK by kynurenine-3monoxygenase (KMO). While KYNA is produced mainly in astrocytes, 3-HK and QUINA are synthesized by activated monocytic cells [16]. 3-HK generates toxic free radicals, leading to neurodegeneration and neuronal apoptosis [69]. Moreover, 3-HK also generates superoxide and hydrogen peroxide promoting oxidative protein damage [25]. In addition to enhanced IDO activity, pro-inflammatory cytokines also enhance the activity of $\mathrm{KMO}$, which in turn enhances 3-HK production and the excitotoxic degenerative arm of the pathway [49]. During a migraine attack, a number of neurobiological changes occur within the neuroinflammation process, such as cytokine-induced stress, oxidative and nitrosative stress caused by peroxynitrates, and release of free radicals throughout arachidonic acid formation. A study demonstrates a significant increase of malondialdehyde plasma levels, the final product of lipid peroxidation, as marker of oxidative stress in migraineurs [70]. The authors suggest a possible novel migraine treatment base on the antioxidant defence system. 3-HK, with its oxidative potential and its enhanced production during inflammation, represents a possible mechanism underlying these observations.

\section{Xanthurenic acid}

Xanthurenic acid (XA), a metabolite of 3-hydroxykynurenine that has been described as a signaling molecule in the mammalian brain, has recently been identified 
Fig. 2 Connection between kynurenine pathway metabolites and known involved pathways in migraine. NMDA-R $N$ methyl-D-aspartate receptor; $\alpha 7$ $n A c h-R$ alpha-7 nicotinic receptor; GPR35 G-proteincoupled receptor $35 ; K Y N A$ kynurenic acid; QUINA quinolinic acid; $C A$ cinnabarinic acid; 3-HK

3-hydroxykynurenine; 3-HANA

3-hydroxyanthranilic acid
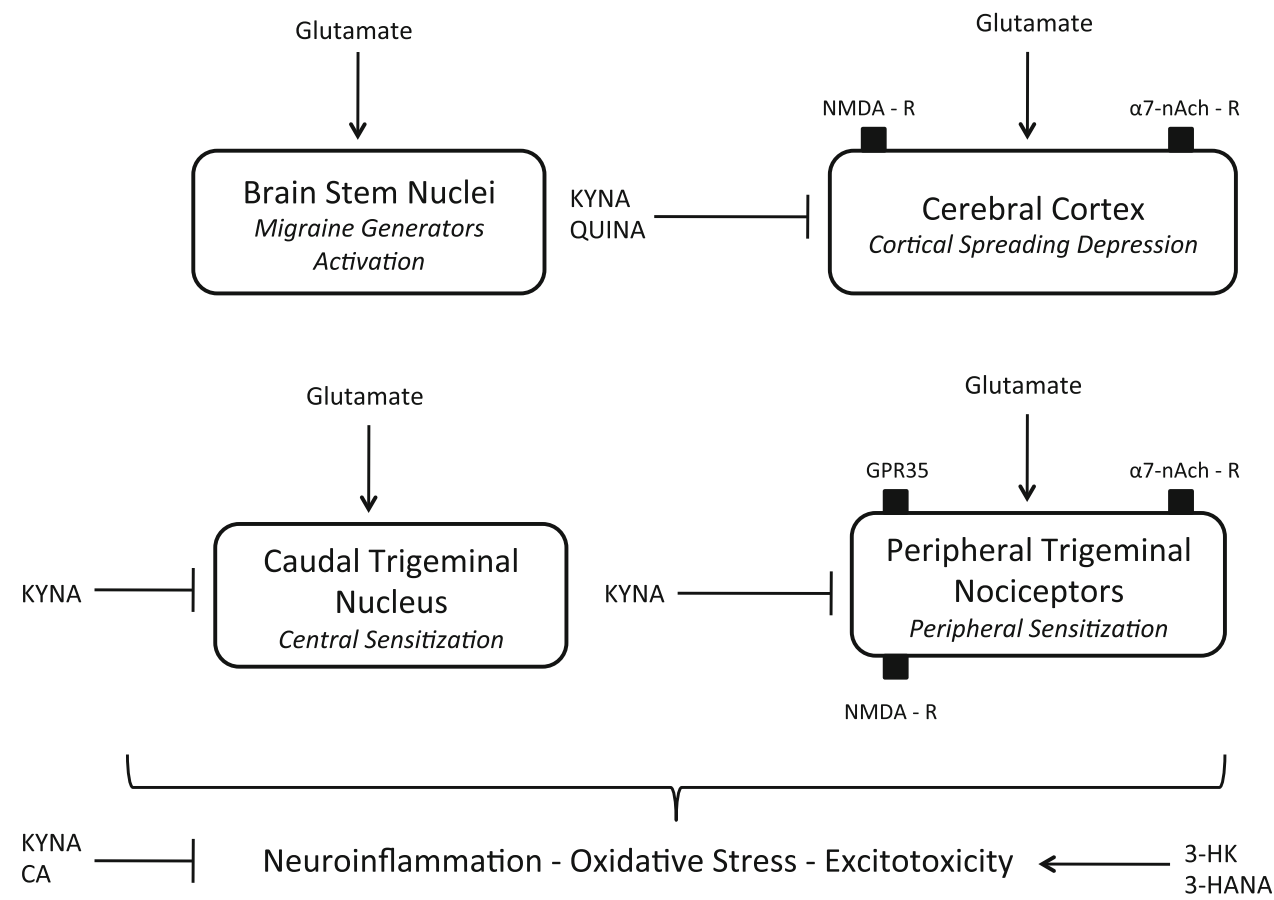

as a selective endogenous group II (mGlu2 and mGlu3) metabotropic glutamate (mGlu) receptor ligand [26, 27]. $\mathrm{XA}$ is formed from $3-\mathrm{HK}$ transamination catalyzed by KAT. This pathway is considered a detoxifying process since it reduces the $3-\mathrm{HK}$ concentration. Moreover, the activation of mGlu3 in mice is required to reduce the NMDA excitotoxic neuronal death, whereas mGlu2 activation may damage neurons exposed to toxic insults [71]. Therefore, the potential neuroprotective role of XA in migraine should be elucidated.

Anthranilic acid and 3-hydroxyanthranilic acid

Anthranilic acid, another by-product of kynurenine metabolism, is virtually an upstream metabolite of KMO, since it is produced from KYNU transamination by kynureninase. 3-HANA can derive either from the hydrolysis of 3-HK or the oxidation of ANA [54]. It auto-oxidizes under physiological conditions, producing hydrogen peroxide and highly reactive hydroxyl radicals [6]. 3-HANA can also cause neuronal damage thus provoking primary or secondary excitotoxicity [72]. 3-HANA has immune-regulatory properties, potentially relevant for autoimmune diseases [73]. The involvement of these metabolites in neuroprotective/neurotoxic processes, and therefore their hypothetical implication in migraine, has not been explored.

Cinnabarinic acid

Cinnabarinic acid (CA) is a by-product of KP, which derives from the condensation of two molecules of
3-HANA. Its biological function is largely unknown [15, 74, 75]. CA harbors two carboxyl groups and a neutral free amino group, which is the basic requirement for an interaction with the orthosteric site of excitatory aminoacid receptors. CA acts as a weak agonist at mGlu4 metabotropic glutamate receptors [15]. CA affords significant protection against NMDA toxicity via activation of mGlu4 [15]. An enhanced formation of CA has been reported during inflammation [15]. Similarly to XA, it may protect neurons against the harmful effect of proinflammatory cytokines or endogenous excitotoxins. Moreover, by its capability to activate mGlu4 receptors and aryl hydrocarbon receptors, CA drives immune systems to an immunosuppressive phenotype [74, 75].

\section{Quinolinic acid}

The observation that intracerebro-ventricular injection of QUINA causes seizure activity in mice gave the first evidence that KP metabolites have central effects [76]. QUINA acts as an orthosteric NMDA receptors with excitatory and neurotoxic properties, resulting in the induction of nitric oxide synthase and excessive nitric oxide-mediated free radical damage [8]. In direct intracerebral administration QUINA leads to neuronal death [77]. In contrast with its glutamatergic properties, QUINA reduces CSD in the rat cortex, probably due to NMDA desensitization (Fig. 2) [78]. Despite of the extended number of studies conducted on QUINA, its role in migraine pathogenesis needs to be further investigated. 


\section{Conclusions}

Migraine possesses one of the highest incidence among all disease affecting the worldwide population [28]. Despite the high number of affected patients and the efforts in pharmacogenetic understanding of this multifactorial disease, pharmacotherapy is still inadequate to mitigate physical sufferings of many migraineurs [79].

The role of glutamate, a major excitatory neurotransmitter in the CNS, is widely recognized in migraine pathogenesis [13]. Critical hallmarks of migraine, such as trigemino-vascular activation, central sensitization and cortical spreading depression involve glutamate. Along one branch of tryptophan metabolism, the kynurenine pathway furnishes a series of compounds able to interact with glutamate receptors, and some of these molecules have recently been linked to migraine [9]. As with the findings obtained in other neurological disorders, where an initial imbalance of a few kynurenine metabolites led to the exploration of the role of the entire pathway in the disease pathophysiology, we suggest that these first studies on kynurenine metabolites in migraine may lead to a wider understanding of its biochemical mechanisms.

A study of the overall scenario of kynurenines in patients suffering from migraine would be helpful to find out alternative options in the research on the etio-pathogenetic mechanisms underlying migraine. Our ongoing studies will point on both the genotypic and the phenotypic profiles of the kynurenine pathway in migraine.

Conflict of interest None.

\section{References}

1. Wirleitner B, Neurauter G, Schröcksnadel K, Frick B, Fuchs D (2003) Interferon-gamma-induced conversion of tryptophan: immunologic and neuropsychiatric aspects. Curr Med Chem 10:1581-1591

2. Bender DA (1983) Biochemistry of tryptophan in health and disease. Mol Aspects Med 6:101-197

3. Magis D, Schoenen J (2011) Treatment of migraine: update on new therapies. Curr Opin Neurol 24(3):203-210

4. Pytliak M, Vargová V, Mechírová V, Felšöci M (2011) Serotonin receptors-from molecular biology to clinical applications. Physiol Res 60(1):15-25

5. Tfelt-Hansen PC, Pihl T, Hougaard A, Mitsikostas DD (2014) Drugs targeting 5-hydroxytryptamine receptors in acute treatments of migraine attacks. A review of new drugs and new administration forms of established drugs. Expert Opin Investig Drugs 23:375-385

6. Schwarcz R, Bruno JP, Muchowski PJ, Wu HQ (2012) Kynurenines in the mammalian brain: when physiology meets pathology. Nat Rev Neurosci 13:465-477

7. Miller CL, Llenos IC, Cwik M, Walkup J, Weis S (2008) Alterations in kynurenine precursor and product levels in schizophrenia and bipolar disorder. Neurochem Int 52:1297-1303
8. Schwarcz MJ, Guillemin GJ, Teipel SJ, Buerger K, Hampel H (2013) Increased 3-hydroxykynurenine serum concentrations differentiate Alzheimer's disease patients from controls. Eur Arch Psychiatry Clin Neurosci 263:345-352

9. Párdutz A, Fejes A, Bohár Z, Tar L, Toldi J, Vécsei L (2012) Kynurenines and headache. J Neural Transm 119:285-296

10. Dahlem MA (2013) Migraine generator network and spreading depression dynamics as neuromodulation targets in episodic migraine. Chaos 23(4):046101

11. Noseda R, Burstein R (2013) Migraine pathophysiology: anatomy of the trigeminovascular pathway and associated neurological symptoms, cortical spreading depression, sensitization, and modulation of pain. Pain 154(Suppl 1):S44-S53

12. Eikermann-Haerter K, Negro A, Ayata C (2012) Spreading depression and the clinical correlates of migraine. Rev Neurosci 24:353-363

13. Vikelis M, Mitsikostas DD (2007) The role of glutamate and its receptors in migraine. CNS Neurol Disord: Drug Targets 6:251-257

14. Takikawa $O$ (2005) Biochemical and medical aspects of indoleamine 2,3-dioxygenase-initiated L-tryptophan metabolism. Biochem Biophys Res Commun 338:12-19

15. Fazio F, Lionetto L, Molinaro G, Bertrand HO, Acherì F, Ngomba RT, Notartomaso S, Curini M, Rosati O, Scarselli P, Di Marco R, Battaglia G, Bruno V, Simmaco M, Pin JP, Nicoletti F, Goudet C (2012) Cinnabarinic acid, an endogenous metabolite of the kynurenine pathway, activates type 4 metabotropic glutamate receptors. Mol Pharmacol 81:643-656

16. Guillemin GJ, Smith DG, Smythe GA, Armati PJ, Brew BJ (2003) Expression of the kynurenine pathway enzymes in human microglia and macrophages. Adv Exp Med Biol 527:105-112

17. Schwarcz R, Pellicciari R (2002) Manipulation of brain kynurenines: glial targets, neuronal effects, and clinical opportunities. J Pharmacol Exp Ther 303:1-10

18. Tavares RG, Tasca CI, Santos CE, Alves LB, Porciuncula LO, Emanuelli T, Souza DO (2002) Quinolinic acid stimulates synaptosomal glutamate release and inhibits glutamate uptake into astrocytes. Neurochem Int 40:621-627

19. Moroni F, Cozzi A, Sili M, Mannaioni G (2012) Kynurenic acid: a metabolite with multiple actions and multiple targets in brain and periphery. J Neural Transm 119:133-139

20. Banerjee J, Alkondon M, Albuquerque EX (2012) Kynurenic acid inhibits glutamatergic transmission to CA1 pyramidal neurons via $\alpha 7 \mathrm{nAChR}$-dependent and -independent mechanisms. Biochem Pharmacol 84:1078-1087

21. Turski WA, Nakamura M, Todd WP, Carpenter BK, Whetsell WO Jr, Schwarcz R (1988) Identification and quantification of kynurenic acid in human brain tissue. Brain Res 454:164-169

22. Stone TW (2000) Development and therapeutic potential of kynurenic acid and kynurenine derivatives for neuroprotection. Trends Pharmacol Sci 21:149-154

23. Eastman CL, Guilarte TR (1990) The role of hydrogen peroxide in the in vitro cytotoxicity of 3-hydroxykynurenine. Neurochem Res 15:1101-1107

24. Guidetti P, Schwarcz R (1999) 3-Hydroxykynurenine potentiates quinolinate but not NMDA toxicity in the rat striatum. Eur $\mathrm{J}$ Neurosci 11:3857-3863

25. Goldstein LE, Leopold MC, Huang X, Atwood CS, Saunders AJ, Hartshorn M, Lim JT, Faget KY, Muffat JA, Scarpa RC, Chylack LT Jr, Bowden EF, Tanzi RE, Bush AI (2000) 3-Hydroxykynurenine and 3-hydroxyanthranilic acid generate hydrogen peroxide and promote alpha-crystallin cross-linking by metal ion reduction. Biochemistry 39:7266-7275

26. Gobaille S, Kemmel V, Brumaru D, Dugave C, Aunis D, Maitre M (2008) Xanthurenic acid distribution, transport, accumulation and release in the rat brain. J Neurochem 105:982-993 
27. Copeland CS, Neale SA, Salt TE (2013) Actions of xanthurenic acid, a putative endogenous group II metabotropic glutamate receptor agonist, on sensory transmission in the thalamus. Neuropharmacology 66:133-142

28. Stovner L, Hagen K, Jensen R, Katsarava Z, Lipton R, Scher A, Steiner T, Zwart JA (2007) The global burden of headache: a documentation of headache prevalence and disability worldwide. Cephalalgia 27:193-210

29. Costa C, Tozzi A, Rainero I, Cupini LM, Calabresi P, Ayata C, Sarchielli P (2013) Cortical spreading depression as a target for anti-migraine agents. J Headache Pain 14:62

30. Burstein R, Yamamura H, Malick A, Strassman AM (1998) Chemical stimulation of the intracranial dura induces enhanced responses to facial stimulation in brain stem trigeminal neurons. J Neurophysiol 79:964-982

31. Bohár Z, Fejes-Szabó A, Tar L, Varga H, Tajti J, Párdutz Á, Vécsei L (2013) Evaluation of c-Fos immunoreactivity in the rat brainstem nuclei relevant in migraine pathogenesis after electrical stimulation of the trigeminal ganglion. Neurol Sci. 34:1597-1604

32. Charles AC, Baca SM (2013) Cortical spreading depression and migraine. Nat Rev Neurol 9:637-644

33. Cananzi AR, D'Andrea G, Perini F, Zamberlan F, Welch KM (1995) Platelet and plasma levels of glutamate and glutamine in migraine with and without aura. Cephalalgia 15:132-135

34. Shimada A, Cairns BE, Vad N, Ulriksen K, Pedersen AM, Svensson P, Baad-Hansen L (2013) Headache and mechanical sensitization of human pericranial muscles after repeated intake of monosodium glutamate (MSG). J Headache Pain 14:2

35. Filipović B, Matak I, Lacković Z (2014) Dural neurogenic inflammation induced by neuropathic pain is specific to cranial region. J Neural Transm 121:555-563

36. Diener HC (2014) CGRP as a new target in prevention and treatment of migraine. Lancet Neurol 13:1065-1067

37. Laursen JC, Cairns BE, Dong XD, Kumar U, Somvanshi RK, Arendt-Nielsen L, Gazerani P (2014) Glutamate dysregulation in the trigeminal ganglion: a novel mechanism for peripheral sensitization of the craniofacial region. Neuroscience 256:23-35

38. Oshinsky ML, Luo J (2006) Neurochemistry of trigeminal activation in an animal model of migraine. Headache 46(Suppl 1):S39-S44

39. Mitsikostas DD, del Sanchez RM, Waeber C, Huang Z, Cutrer FM, Moskowitz MA (1999) Non-NMDA glutamate receptors modulate capsaicin induced c-fos expression within trigeminal nucleus caudalis. Br J Pharmacol 127:623-630

40. McGehee DS, Heath MJ, Gelber S, Devay P, Role LW (1995) Nicotine enhancement of fast excitatory synaptic transmission in CNS by presynaptic receptors. Science 269:1692-1696

41. Tajti J, Szok D, Párdutz Á, Tuka B, Csáti A, Kuris A, Toldi J, Vécsei L (2012) Where does a migraine attack originate? In the brainstem. J Neural Transm 119(5):557-568

42. Weiller C, May A, Limmroth V, Juptner M, Kaube H, Schayck RV, Coenen HH, Diener HC (1995) Brain stem activation in spontaneous human migraine attacks. Nat Med 1:658-660

43. Welch KM, Nagesh V, Aurora SK, Gelman N (2001) Periaqueductal gray matter dysfunction in migraine: cause or the burden of illness? Headache 41:629-637

44. Renno WM, Alkhalaf M, Mousa A, Kanaan RA (2008) A comparative study of excitatory and inhibitory amino acids in three different brainstem nuclei. Neurochem Res 33(1):150-159

45. Bolay H, Reuter U, Dunn AK, Huang Z, Boas DA, Moskowitz MA (2002) Intrinsic brain activity triggers trigeminal meningeal afferents in a migraine model. Nat Med 8:136-142

46. Ayata C, Moskowitz MA (2006) Cortical spreading depression confounds concentration-dependent pial arteriolar dilation during
$N$-methyl-D-aspartate superfusion. Am J Physiol Heart Circ Physiol 290:H1837-H1841

47. Costa C, Tozzi A, Rainero I, Cupini LM, Calabresi P, Ayata C, Sarchielli P (2013) Cortical spreading depression as a target for anti-migraine agents. J Headache Pain 23(14):62

48. Vécsei L, Szalárdy L, Fülöp F, Toldi J (2013) Kynurenines in the CNS: recent advances and new questions. Nat Rev Drug Discov 12:64-82

49. Myint AM, Schwarz MJ, Verkerk R, Mueller HH, Zach J, Scharpé S, Steinbusch HW, Leonard BE, Kim YK (2011) Reversal of imbalance between kynurenic acid and 3-hydroxykynurenine by antipsychotics in medication-naïve and medication-free schizophrenic patients. Brain Behav Immun 25:1576-1581

50. Martelletti P, Stirparo G, Morrone S, Rinaldi C, Giacovazzo M (1997) Inhibition of intercellular adhesion molecule-1 (ICAM-1), soluble ICAM-1 and interleukin-4 by nitric oxide expression in migraine patients. J Mol Med (Berl) 75:448-453

51. Munno I, Marinaro M, Bassi A, Cassiano MA, Causarano V, Centonze V (2001) Immunological aspects in migraine: increase of IL-10 plasma levels during attack. Headache 41:764-767

52. Drummond PD (2006) Tryptophan depletion increases nausea, headache and photophobia in migraine sufferers. Cephalalgia 26:1225-1233

53. Wang Y, Liu H, McKenzie G, Witting PK, Stasch JP, Hahn M, Changsirivathanathamrong $\mathrm{D}, \mathrm{Wu} \mathrm{BJ}$, Ball HJ, Thomas SR, Kapoor V, Celermajer DS, Mellor AL, Keaney JF Jr, Hunt NH, Stocker R (2010) Kynurenine is an endothelium-derived relaxing factor produced during inflammation. Nat Med 16:279-285

54. Chen Y, Guillemin GJ (2009) Kynurenine pathway metabolites in humans: disease and healthy States. Int J Tryptophan Res 2:1-19

55. Albuquerque EX, Schwarcz R (2013) Kynurenic acid as an antagonist of $\alpha 7$ nicotinic acetylcholine receptors in the brain: facts and challenges. Biochem Pharmacol 85:1027-1032

56. Guidetti P, Amori L, Sapko MT, Okuno E, Schwarcz R (2007) Mitochondrial aspartate aminotransferase: a third kynurenateproducing enzyme in the mammalian brain. J Neurochem 102:103-111

57. Oláh G, Herédi J, Menyhárt A, Czinege Z, Nagy D, Fuzik J, Kocsis K, Knapp L, Krucsó E, Gellért L, Kis Z, Farkas T, Fülöp F, Párdutz A, Tajti J, Vécsei L, Toldi J (2013) Unexpected effects of peripherally administered kynurenic acid on cortical spreading depression and related blood-brain barrier permeability. Drug Des Devel Ther 16(7):981-987

58. Kiss C, Shepard PD, Bari F, Schwarcz R (2004) Cortical spreading depression augments kynurenate levels and reduces malonate toxicity in the rat cortex. Brain Res 1002:129-135

59. Chauvel V, Vamos E, Pardutz A, Vecsei L, Schoenen J, Multon S (2012) Effect of systemic kynurenine on cortical spreading depression and its modulation by sex hormones in rat. Exp Neurol 236:207-214

60. Knyihár-Csillik E, Toldi J, Mihály A, Krisztin-Péva B, Chadaide Z, Németh H, Fenyo R, Vécsei L (2007) Kynurenine in combination with probenecid mitigates the stimulation-induced increase of c-fos immunoreactivity of the rat caudal trigeminal nucleus in an experimental migraine model. J Neural Transm 114:417-421

61. Vámos E, Párdutz A, Varga H, Bohár Z, Tajti J, Fülöp F, Toldi J, Vécsei L (2009) 1-Kynurenine combined with probenecid and the novel synthetic kynurenic acid derivative attenuate nitroglycerininduced nNOS in the rat caudal trigeminal nucleus. Neuropharmacology 57:425-429

62. Vámos E, Fejes A, Koch J, Tajti J, Fülöp F, Toldi J, Párdutz A, Vécsei L (2010) Kynurenate derivative attenuates the nitroglycerin-induced CamKII $\alpha$ and CGRP expression changes. Headache $50: 834-843$ 
63. Zhang YQ, Ji GC, Wu GC, Zhao ZQ (2003) Kynurenic acid enhances electroacupuncture analgesia in normal and carrageenan-injected rats. Brain Res 966:300-307

64. Mecs L, Tuboly G, Nagy E, Benedek G, Horvath G (2009) The peripheral antinociceptive effects of endomorphin-1 and kynurenic acid in the rat inflamed joint model. Anesth Analg 109:1297-1304

65. Knyihár-Csillik E, Chadaide Z, Okuno E, Krisztin-Péva B, Toldi J, Varga C, Molnár A, Csillik B, Vécsei L (2004) Kynurenine aminotransferase in the supratentorial dura mater of the rat: effect of stimulation of the trigeminal ganglion. Exp Neurol $186: 242-247$

66. Wang J, Simonavicius N, Wu X, Swaminath G, Reagan J, Tian H, Ling L (2006) Kynurenic acid as a ligand for orphan G proteincoupled receptor GPR35. J Biol Chem 281:22021-22028

67. Fejes-Szabó A, Bohár Z, Vámos E, Nagy-Grócz G, Tar L, Veres G, Zádori D, Szentirmai M, Tajti J, Szatmári I, Fülöp F, Toldi J, Párdutz Á, Vécsei L (2014) Pre-treatment with new kynurenic acid amide dose-dependently prevents the nitroglycerine-induced neuronal activation and sensitization in cervical part of trigemino-cervical complex. J Neural Transm 121:725-738

68. Lugo-Huitrón R, Blanco-Ayala T, Ugalde-Muñiz P, CarrilloMora P, Pedraza-Chaverrí J, Silva-Adaya D, Maldonado PD, Torres I, Pinzón E, Ortiz-Islas E, López T, García E, Pineda B, Torres-Ramos M, Santamaría A, La Cruz VP (2011) On the antioxidant properties of kynurenic acid: free radical scavenging activity and inhibition of oxidative stress. Neurotoxicol Teratol 33:538-547

69. Chiarugi A, Calvani M, Meli E, Traggiai E, Moroni F (2001) Synthesis and release of neurotoxic kynurenine metabolites by human monocyte-derived macrophages. J Neuroimmunol 120:190-198

70. Gupta R, Pathak R, Bhatia MS, Banerjee BD (2009) Comparison of oxidative stress among migraineurs, tension-type headache subjects, and a control group. Ann Indian Acad Neurol $12: 167-172$
71. Corti C, Battaglia G, Molinaro G, Riozzi B, Pittaluga A, Corsi M, Mugnaini M, Nicoletti F, Bruno V (2007) The use of knock-out mice unravels distinct roles for mGlu2 and mGlu3 metabotropic glutamate receptors in mechanisms of neurodegeneration/neuroprotection. J Neurosci 27:8297-8308

72. Fejes A, Párdutz A, Toldi J, Vécsei L (2011) Kynurenine metabolites and migraine: experimental studies and therapeutic perspectives. Curr Neuropharmacol 9:376-387

73. Fallarino F, Grohmann U, Vacca C, Bianchi R, Orabona C, Spreca A, Fioretti MC, Puccetti P (2002) T cell apoptosis by tryptophan catabolism. Cell Death Differ 9:1069-1077

74. Lowe MM, Mold JE, Kanwar B, Huang Y, Louie A, Pollastri MP, Wang C, Patel G, Franks DG, Schlezinger J, Sherr DH, Silverstone AE, Hahn ME, McCune JM (2014) Identification of cinnabarinic acid as a novel endogenous aryl hydrocarbon receptor ligand that drives IL-22 production. PLoS ONE 9:e87877

75. Fazio F, Zappulla C, Notartomaso S, Busceti C, Bessede A, Scarselli P, Vacca C, Gargaro M, Volpi C, Allegrucci M, Lionetto L, Simmaco M, Belladonna ML, Nicoletti F, Fallarino F (2014) Cinnabarinic acid, an endogenous agonist of type-4 metabotropic glutamate receptor, suppresses experimental autoimmune encephalomyelitis in mice. Neuropharmacology $81: 237-243$

76. Lapin IP (1978) Stimulant and convulsive effects of kynurenines injected into brain ventricles in mice. J Neural Transm 42:37-43

77. Schwarcz R, Whetsell WO Jr, Mangano RM (1983) Quinolinic acid: an endogenous metabolite that produces axon-sparing lesions in rat brain. Science 219:316-318

78. Obrenovitch TP, Urenjak J (2003) Accumulation of quinolinic acid with neuroinflammation: does it mean excitotoxicity? Adv Exp Med Biol 527:147-154

79. Gentile G, Chiossi L, Lionetto L, Martelletti P, Borro M (2014) Pharmacogenetic insights into migraine treatment in children. Pharmacogenomics 15:1539-1550 